\title{
Challenges of Massive Access in Highly-Dense LTE-Advanced Networks with Machine-to-Machine Communications
}

\author{
Kan Zheng Senior Member, IEEE ${ }^{\dagger}$, Suling Ou ${ }^{\dagger}$, Jesus Alonso-Zarate Senior Member, IEEE* ${ }^{*}$ Mischa \\ Dohler Fellow, IEEE**, Fei Liu* and Hua $\mathrm{Zhu}^{\dagger}$ \\ ${ }^{\dagger}$ Wireless Signal Processing and Network Lab \\ Key laboratory of Universal Wireless Communication, Ministry of Education \\ Beijing University of Posts \& Telecommunications \\ Beijing, China, 100088 \\ * Centre Tecnologic de Telecommunicacions de Catalunya (CTTC) \\ Barcelona, Spain, 08860 \\ ** King's College London (KCL) \\ * Aachen University, Germany \\ Contact email: kzheng@ieee.org
}

Correspondence: Prof. Kan Zheng

Wireless Signal Processing and Network Lab

Beijing University of Posts \& Telecommunications

P.O.Box 93, No. 10, Xi Tu Cheng Road, Beijing, China

Phone: (86) 10-62282245, Fax: (86) 10-62282921

Email: kzheng@ieee.org 


\title{
Challenges of Massive Access in Highly-Dense LTE-Advanced Networks with Machine-to-Machine Communications
}

\author{
Kan Zheng Senior Member, IEEE ${ }^{\dagger}$, Suling $\mathrm{Ou}^{\dagger}$, \\ Jesus Alonso-Zarate Senior Member, IEEE*, Mischa Dohler Fellow, IEEE**, Fei Liu* and Hua Zhu ${ }^{\dagger}$ \\ ${ }^{\dagger}$ Wireless Signal Processing and Network Lab \\ Key laboratory of Universal Wireless Communication, Ministry of Education \\ Beijing University of Posts \& Telecommunications \\ Beijing, China, 100088 \\ * Centre Tecnologic de Telecommunicacions de Catalunya (CTTC) \\ Barcelona, Spain, 08860 \\ ** King's College London (KCL) \\ * Aachen University, Germany \\ Contact email: kzheng@ieee.org
}

\begin{abstract}
Machine-to-Machine (M2M) wireless systems are being standardized to provide ubiquitous connectivity between machines without the need of human intervention. A natural concern of cellular operators and service providers is the impact that these machine type communications (MTC) will have onto the current human type communications (HTC). Given the exponential growth of MTC traffic, it is utmost important to ensure that current voice and data traffic is not jeopardized. This paper investigates the limits of MTC traffic coexisting with HTC traffic in LTE-A networks, such that the human customer churn is minimized. We show that, under proper design, the outage probability of HTC is marginally impacted whilst duty cycle and access delay of MTC are reasonably bounded to ensure viable M2M operations.
\end{abstract}

Index Terms- M2M, LTE, Radio Access 


\section{INTRODUCTION}

Current market penetration and recent predictions confirm that machine-to-machine (M2M) system deployments are increasing exponentially. This is driven by the needs of industries to automate their real-time monitoring and control processes and the increasing popularity of smart applications to improve human well-being. Examples are the automotive industry, which utilizes sensors to monitor the status of critical car components; the smart grid industry, which monitors critical points in the power transportation and distribution networks; or the smart city market, which provides innovative services to the citizens by using real-time sensory data from the streets.

Capitalizing on the potentials and applicability of M2M, various industrial standardization bodies have commenced embedding the unique needs of M2M systems into legacy wireless communication systems. This is the case of the IEEE and the IETF, which are defining standards suitable for M2M applications based on the short and mid-range technologies such as the IEEE 802.11 or the IEEE 802.15.4x Standards, with different amendments for low-power industrial and smart applications (e.g., smart cities and smart grids). However, such networks suffer from some limitations, such as the use of shared exempt frequency channels (thus prone to interference) and limited radio coverage, which compromises the mass deployment of M2M services, but is easily overcome by cellular networks. Indeed, cellular networks constitute a very interesting alternative to providing $\mathrm{M} 2 \mathrm{M}$ coverage. The fact that the infrastructure is already installed enables a fast and low-cost deployment of M2M services in very short time, also providing a simple network topology based on one-hop communications.

Unfortunately, current 3G and LTE (Long Term Evolution) cellular networks have not been designed for M2M traffic. Instead, they were mainly designed to support human-based services such as voice or webbrowsing and bandwidth demanding services such as video streaming. Therefore, the network architecture needs to be improved to accommodate new M2M service requirements without sacrificing the quality of human-based services. For example, among others, M2M data traffic is mainly uplink while current data traffic in human-based applications is mainly downlink; the amount of data per device is very small (e.g., only few bits of information per transaction); the number of envisioned devices in the network is very high, orders of magnitude above the amount of humans; also, ultra-high energy efficiency is necessary to ensure the long life-time of networks once deployed to make sure that M2M deployments can be 
autonomous and do not require frequent human intervention.

For these reasons, leading standardization bodies, such as the International Telecommunications Union (ITU), the European Telecommunications Standards Institute (ETSI), the Third Generation Partnership Project (3GPP), the Telecommunications Industry Association (TIA), or the Chinese Communications Standard Association (CCSA), and global initiatives such as the OneM2M, have commenced work on satisfying these and other constraints whilst not jeopardizing current cellular system usage for humanbased applications. For example, ETSI launched in 2009 an M2M technical committee which actively looks into architectural design; whilst 3GPP is incorporating M2M through its Machine Type Communications (MTC) designs, in coexistence with Human Traffic Communications (HTC). In all cases, one of the main objectives of these organizations is to identify open challenges where efficient solutions can be proposed.

Among others, the Random Access Channel (RACH) of LTE (and LTE-A, which is essentially the same at the RACH level) has been identified as a key area where improvements for MTC traffic are needed. The fact that the RACH of LTE is (still) based on a random-access mechanism turns it into a potential bottleneck for the performance of cellular networks if the number of MTC devices grows [1]. For this reason, the RACH of LTE has attracted lots of attention from the research community. However, with the exception of [4], research related to the co-existence of MTC and HTC devices, which is the expected modus operandi in practical networks, has been scarce to date. This is the motivation for the work presented in this paper, where we address this uncertainty and show, via computer-based simulations, that the current operation of the RACH of LTE is suitable for the projected M2M traffic requirements as long as appropriate priority mechanisms are applied to balance MTC and HTC traffic. A major challenge has been to ensure that designed coexistence mechanisms are 3GPP-compliant and thus practically viable.

To this end, in Section II, we overview latest 3GPP standardization activities and describe in sufficient detail the access method of the RACH of LTE/LTE-A. In Section III, we introduce two access methods based on weighting the access priority of HTC and MTC according to the service types, and show that the impact of MTC onto HTC heavily depends on the access method of choice. Based on these results, we propose two improved access mechanisms to reduce the impact of MTC onto HTC. We have then simulated the RACH of LTE with the proposed schemes and thoroughly discuss the numerical results in Section IV. Simulations confirm that, under suitable design, the impact of MTC onto HTC can remain almost 
negligible, even for a very large number of M2M devices in the same cellular coverage cell. Furthermore, we show that relevant performance metrics, such as average access delay, energy consumption, and outage probability (i.e., probability of not getting access to the network) remain reasonably bounded to facilitate delay-critical M2M applications. Finally, conclusions are drawn and future challenges outlined in Section $\mathrm{V}$.

\section{3GPP LTE-A MTC STANDARD AND RACH PROCEDURES}

3GPP is focused on the definition of technical requirements and functional specifications aimed at two different objectives; i) to adapt existing technologies ( $2 \mathrm{G}$ and $3 \mathrm{G}$ networks) to support MTC traffic, and ii) to optimize the design of future networks (LTE and LTE-A) to provide efficient MTC services [10]. From Release 10 onwards, 3GPP started to work in the design of a suitable core network architecture (from the application to the devices), services, specific signaling reduction and optimization at the radio access network (RAN) for MTC services, including mechanisms for identifying and addressing devices, device triggering, and usage of SMS over packet switching connections for data transfer. For Release 12 and 13, the work is being mainly focused on optimization of transmissions for small data packets, triggering of devices with different priority, higher energy efficiency for terminals, and group management functions. The addition of new interfaces in the network architecture is also being designed to decouple MTC servers from the overall network functional blocks to ensure that HTC and MTC traffic can be handled simultaneously in an efficient manner. 3GPP takes into account the specific requirements posed by a huge variety of different MTC services which, besides posing very diverse Quality of Service (QoS) requirements, may also need to handle a huge amount of devices, provide ultra-low energy-consumption and low-cost solutions, and facilitate the coexistence of both MTC and HTC applications.

The work presented in this paper focuses on the RACH of the LTE/LTE-A standards and contributes to the need to evaluate the impact of MTC traffic on the requirements of HTC users. According to the ETSI M2M architecture and the network improvements for MTC developed by the 3GPP [11], [12], exemplified in Fig.1, three types of MTC access methods are defined:

- Direct Access: An MTC can directly access an Evolved NodeB (eNB) in cellular networks without any intermediate device. This type of communication is similar to that of a regular User Equipment 


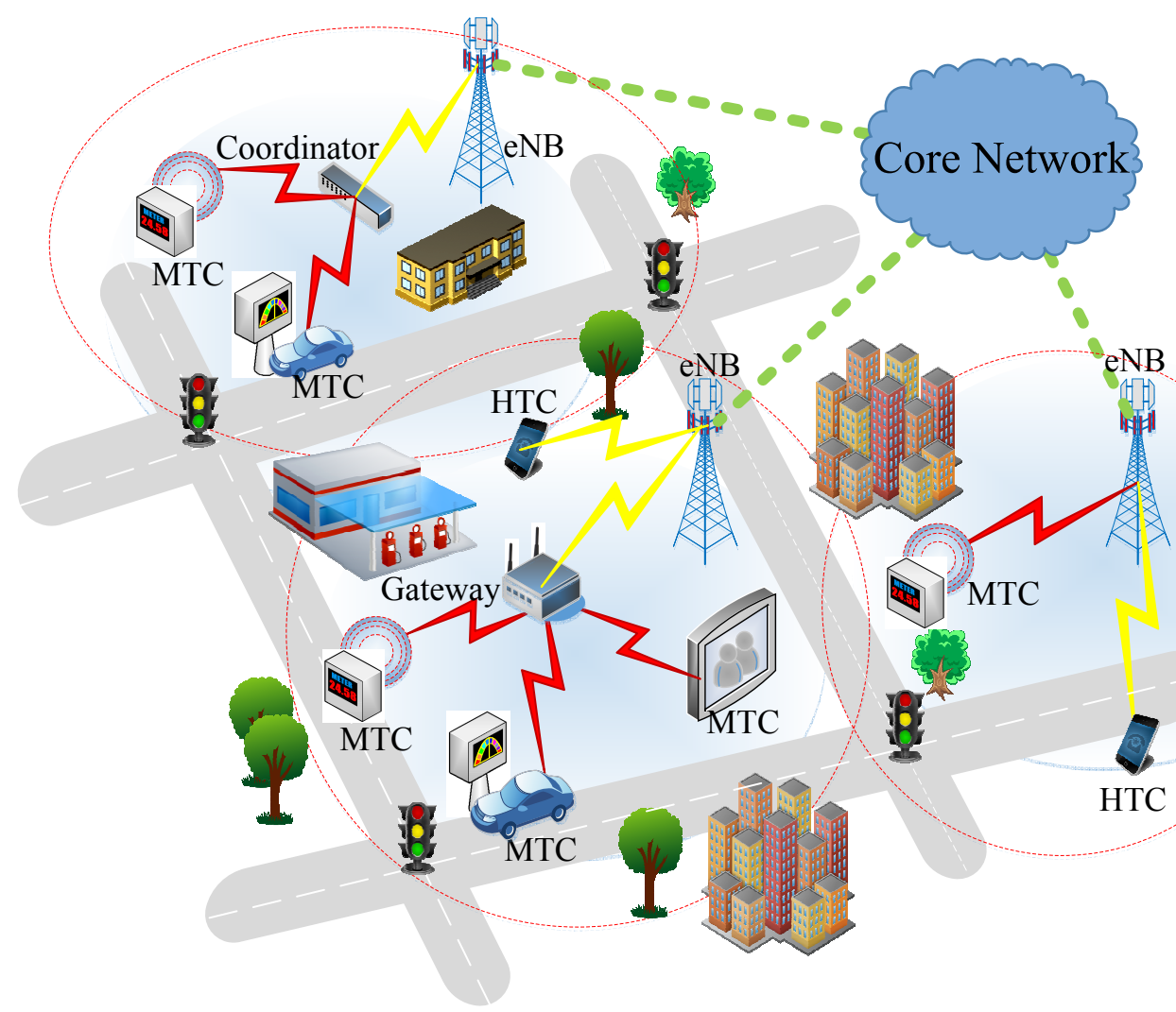

Fig. 1. High-level illustration of cellular networks with MTC and HTC devices co-existing [9].

(UE). Even though this is the most simple access method, it may lead to traffic congestion in the presence of a huge number of MTC devices in the network if the amount of access resources is not enough.

- Gateway Access: MTC devices can obtain cellular connectivity through M2M gateways. An M2M gateway is a dedicated device with different functionalities from those of regular MTC devices. M2M gateways relay data transmissions between the eNB and a group of MTC devices, but do not generate own data traffic.

- Coordinator Access: In some cases, MTCs can also play the role of coordinators to help their neighbor MTC devices to access the network by providing a two-hop communication. Adjacent MTC devices can be grouped before transmission in order to reduce redundant signaling and avoid congestion. One MTC device in the group can be assigned the coordinator role to communicate with the eNB, thus acting as temporary M2M gateway to transmit the data from all the members of its group. This access method can reduce the overall power consumption of all MTCs and thus extend the service life of the battery-constrained devices. 


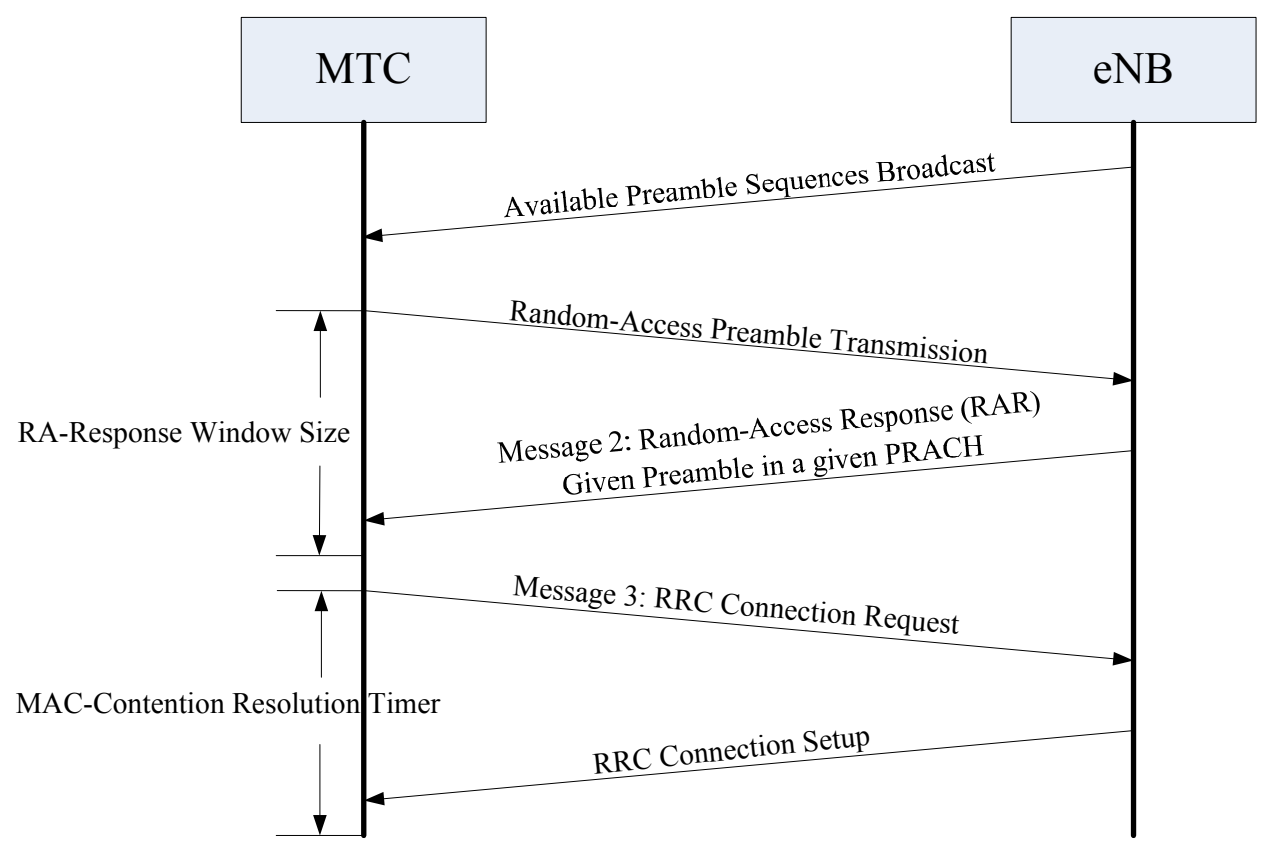

Fig. 2. Random access procedure in LTE/LTE-A networks [14].

All HTCs and MTCs are provided services by the eNBs either directly or indirectly. From the view of network, the co-existence between HTCs and MTCs raises the problem of radio resource competition to each other in the same network.

Regardless of whether the entity requesting access to the cellular network is an MTC device, a dedicated M2M gateway, or a coordinator MTC device, it is necessary to establish a Radio Resource Control (RRC) connection with the eNB in order to be able to transmit data. To do so, in LTE and LTE-A, a periodic amount of frequency-time resources are allocated for the Physical Random Access Channel (PRACH), through which RRC connections can be established [13]. Devices are informed of the available PRACH resources with the system information broadcasted by the eNB in either the broadcast or other downlink control channels. When a device attempts to connect to the eNB, the random access procedure comprises the exchange of the following four messages, which are also summarized in Fig. 2:

- Message 1 - Random-Access Preamble Transmission: A device attempting to establish a connection transmits a randomly selected preamble (chosen among a set of a maximum of 64 possible preambles, even though some of them may be reserved for prioritized access) in the next available RACH resource. The eNB can estimate the transmission time of the device by detecting its random-access preamble. Since it is possible that multiple devices send preambles simultaneously, there may be 
collisions during the access procedure. These collisions will be detected in Message 3, as explained later.

- Message 2 - Random-Access Response (RAR): For each detected preamble in each access resource (slot), the eNB sends a time advance command to all the devices which transmitted a specific preamble in a specific PRACH to adjust synchronization. In addition, the eNB allocates transmission resources to the devices which sent a given preamble in a given PRACH for the transmission of Message 3. If a device sends Message 1 and it does not receive the RAR from the eNB in a certain period of time, called RA-Response Window Size, or it receives a RAR which does not attach information related to its access request, it postpones the access attempt to the next RACH opportunity. This can happen due to either a collision or a channel fading which has corrupted the transmitted RAR. The postponement periods are determined by the backoff parameters indicated. For example, upon a failure in the first attempt, the value of the backoff index is 1 and the slot duration lasts $10 \mathrm{~ms}$. Therefore, the device will attempt new access in a random time between 0 and $10 \mathrm{~ms}$.

- Message 3 - Radio Resource Control (RRC) Connection Request: The device which sent Message 1 to initiate the access procedure and receives the RAR associated to its transmitted preamble transmits, in the assigned resources notified in the RAR, its temporary terminal identity to the eNB using the Physical Uplink Shared Channel (PUSCH) to request an RRC connection. In the case that two or more devices sent Message 1 using the same preamble in the same PRACH, and the collision was not detected in Message 2 (due to a constructive interference), Message 3, will collide. To detect such collision, Message 3 is transmitted with Hybrid Automatic Repeat-reQuest (ARQ). Upon a maximum number of attempts to transmit Message 3, a collision is declared, and access to the system is postponed.

- Message 4 - RRC Connection Setup: The eNB sends information allocating resources to each of the devices which gained access, specifying their addresses. Therefore, the connection is established and the device can start the transmission of data.

\section{IMPROVING THE RACH FOR MTC AND HTC}

As it has been described in the previous section, the RACH for LTE and LTE-A is based on randombased contention access. Note that devices select a preamble according to the given rule and transmit in 
TABLE I

COMPARISON OF MTC ACCESS METHODS.

\begin{tabular}{|c|c|c|c|c|}
\hline Name & Evaluation Quality & Inter-Cell Interference & HTC Impact & Coordinator or Gateway \\
\hline ES-MACPA [3] & Energy Consumption & No & No & Yes \\
\hline K-means-based [4] & Energy Consumption & No & Yes & Nes \\
\hline RA preambles separation [6] & Throughput & No & No & No \\
\hline Massive Access Management [7] & Jitter & No & No & No \\
\hline Cooperative ACB [8] & Delay and Throughput & Multi-cell and Pico & No & No \\
\hline Multi-Group Random Access [9] & Signal-to-Interference-Ratio & Multi-cell & &
\end{tabular}

a slot, expecting to have not collided with any other device. Indeed, the operation of the RA is based on a variation of Frame Slotted ALOHA, which suffers from congestion when the number of contending devices is very high, as the probability of collision grows exponentially with the number of devices. Therefore, in the presence of a high number of MTC devices especially with direct access, the access performance of HTC devices will be degraded, thus jeopardizing human-based applications.

To deal with this limitation, a lot of works on the RACH in LTE with M2M are being carried out. Some of the most relevant contributions are summarized in Table I and a comprehensive survey can be found in [16]. Most of these works focus on the optimization of existing networks for M2M traffic in isolated conditions, i.e., without HTC traffic into consideration. For example, the work in [2] provides an overview of the network architecture and features of MTC in the 3GPP network and evaluates the performance of MTC using various access schemes in terms of average access delay, throughput, jitter, and energy efficiency. Other works focus on controlling inter-cell interference when the number of MTC devices is very high [3]- [7] and the QoS requirements for different applications need to smoothly coexist $[8]$.

In this paper, we propose splitting the devices of a network into two groups, i.e., one with all the MTC devices and another one with all the HTC devices. Then, we study two backwards compatible ways of providing each of these groups with access priority against the other group. Namely,

1) Priority HTC (PHTC): HTC is always prioritized against MTC in the case of collision.

2) Priority MTC (PMTC): MTC is always prioritized against HTC in the case of collision.

The eNB can distinguish between HTCs and MTCs through their terminal identities contained in Message 3 of the random access procedure (see Section III for more details), or by applying a barrier 
mechanism, i.e., using different set of pre-defined preambles for each group. Priority is granted in Message 4, when resources for transmission are granted. If there are multiple devices of one kind requesting access together, the one with largest backoff time will be granted access first. The backoff time of each device is recorded during the random access procedure and is used to determine the access priorities

These two priority classes embody two extremes, i.e., either gives full priority to human or to machineto-machine traffic. For practical rollouts, a weighted priority approach could be envisaged; however, according to the performance insights presented in the next section, this would not be needed.

\section{Performance And Discussions}

In this section, the performance of the two methods defined in the previous section is evaluated by means of computer-based simulations based on MATLAB software, where the operation of the RACH of LTE has been implemented. The focus is therefore on the RACH and not on actual data transmission.

The considered key performance indicators (KPIs) are: $i$ ) the blocking probability, $i i$ ) the average access delay, and iii) the achievable duty cycle of devices when using the PHTC and PMTC techniques presented in the previous section. All these parameters are defined in the next subsection.

\section{A. Definitions}

The blocking probability is defined as the ratio of devices failing to access the eNB due to exceeding their maximum allowed access delay to the total number of devices attempting to get access.

The average access delay is defined as the average time elapsed from the moment when a device sends the first access request until the moment when it succeeds and radio resources are allocated for transmission.

The duty-cycle is defined as the ratio between the device active time (when the radio frontend is on) to the device sleeping time (when the radio frontend is off) and can be expressed as

$$
\rho=\frac{\left(P_{B} T_{M A X}\right)+\left(1-P_{B}\right) T_{\text {access }}}{T_{I N}},
$$

where $P_{B}$ is the blocking probability, $T_{M A X}$ is the maximum access delay in the case that the access is 
TABLE II

SIMULATION PARAMETERS FOR THE RACH OF LTE [15]

\begin{tabular}{|c|c|c|}
\hline Parameter & & Value \\
\hline RA Transmission Time Interval (TTI) & \multicolumn{2}{|c|}{$20 \mathrm{sf}^{-1}=20 \mathrm{~ms}$} \\
\hline $\multirow[t]{2}{*}{$ Back index }$=\{$ BackoffParameter $\}$ & $\{0, \ldots, 12$ & $10,20, \ldots, 480,960\} \mathrm{ms}$ \\
\hline & HTC & MTC \\
\hline Number of Devices & 100 & $100 \sim 60000$ \\
\hline Inter-Arrival Rate (access requests/second) - Poisson Distribution & $1 / 300$ & $1 / 900$ and $1 / 1200$ \\
\hline Maximum Allowed Access Delay & $100 \mathrm{~ms}$ & $1000 \mathrm{~ms}$ \\
\hline
\end{tabular}

dismissed, $T_{\text {access }}$ is the actual access delay in the case of successful access, and $T_{I N}$ is the inter-arrival time, i.e., the time between two consecutive access requests.

\section{B. Scenario}

In order to evaluate the performance of the two techniques, PMTC and PHTC, in terms of the KPI defined above, we consider a single-cell cellular LTE network formed by 100 HTC devices and a number of MTC devices which has been varied from 100 to 60,000 .

In order to focus on the performance of the contention process of the RACH, ideal wireless channel conditions have been assumed. This means that an access request can only fail due to collisions with other devices, and not due to channel fading. This suits this study which focuses on a congested network. The values of the parameters used to simulate the RACH of LTE are listed in Table II. Two typical scenarios are considered where different average access interval are assumed, i.e., 20 minutes for Scenario 1 and 15 minutes for Scenario 2.

\section{Results}

Fig. 3(a) shows the blocking probability of HTC and MTC when using PHTC and PMTC as a function of the number of MTC devices in the network. For the PMTC methods, the blocking probability of HTC traffic seriously increases with the number of devices. It is worth observing that the difference between the two methods for MTC devices is very small. This means that MTC traffic is slightly impacted by the applied priority method. MTC devices are the ones causing the congestion of the network, and thus the treatment applied to HTC has little impact on the performance perceived by MTC devices.

Therefore, a network operator should always give highest priority to HTC in order to ensure good 


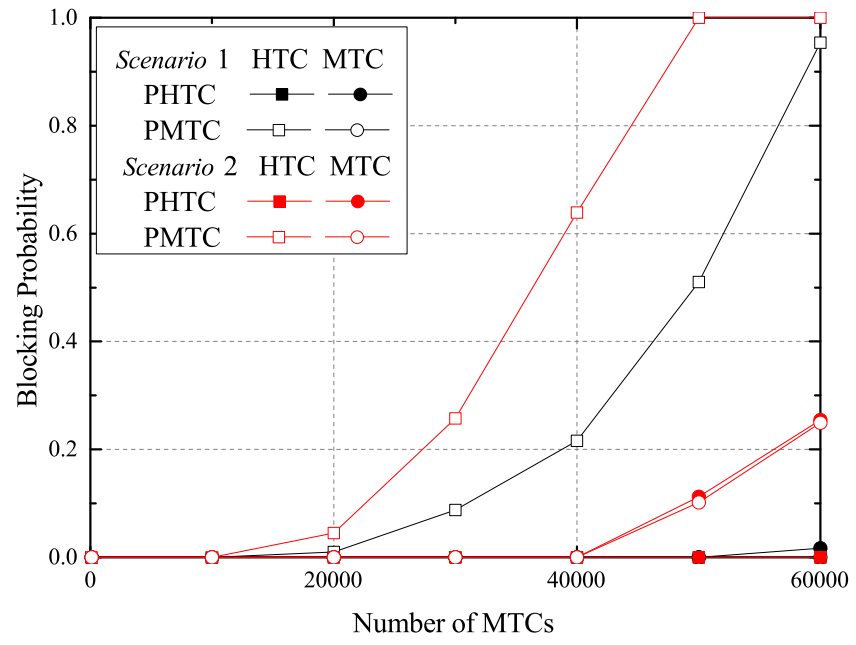

(a) Blocking Probability.

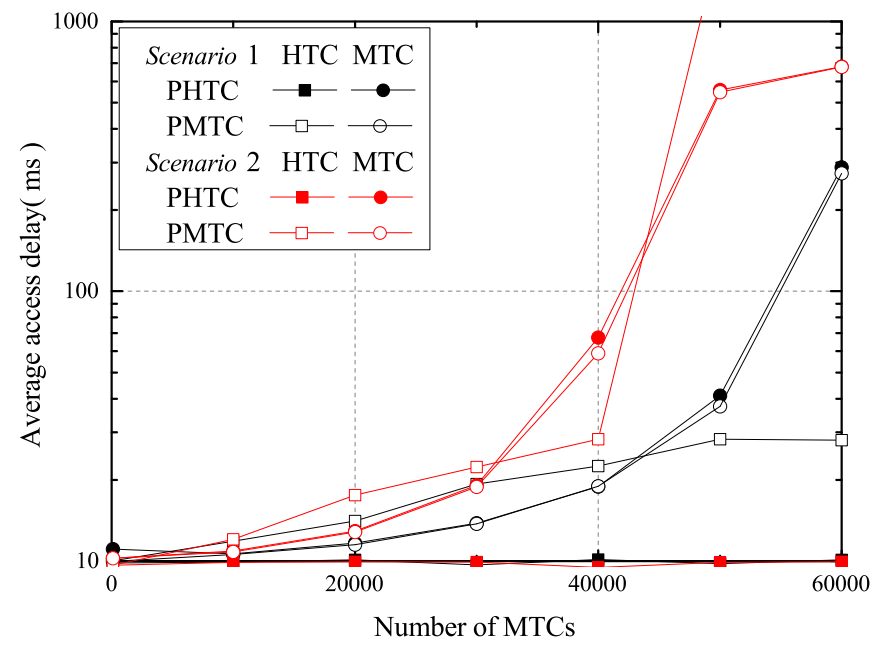

(b) Average Access Delay.

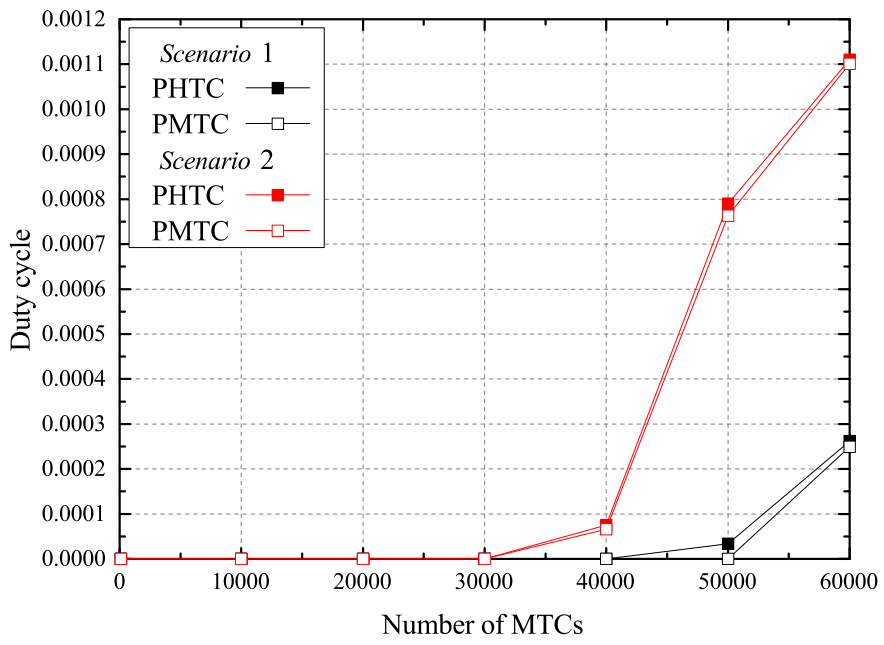

(c) Duty Cycle of MTC Devices.

Fig. 3. Performance of HTC and MTC Devices under Two Scenarios. 
service for HTC whilst only marginally jeopardizing MTC. For delay-constrained M2M applications, a new group of MTCs, e.g., MTC-class II, could be defined with the same treatment as HTC devices, so that their performance does not suffer from congestion when the number of devices is very high.

The average access delay of HTC and MTC devices is shown in Fig. 3(b) for both methods. In all cases, this value increases with the number of contending MTC devices. In addition, and as it could be expected, with the PMTC method, the average access delay of HTC devices is higher than that with PHTC. This difference becomes less remarkable for the average access delay of MTC devices, in which case the two lines overlap, showing the same result in terms of average delay. Again, these results confirm that giving priority to HTC marginally impacts MTC, and not the other way around. The value of the achievable duty-cycle for MTC devices is shown in Fig. 3(c). Results show that the duty-cycle increases with the number of contending devices and, as in the case of the blocking probability and the average delay, this trend holds regardless of the priority method used.

The value of the duty-cycle has a direct impact on the energy consumption of the devices and thus the lifetime of the network. Lower values of the duty cycle lead to lower power consumption and thus longer lifetimes. Let us assume for example that an MTC device consumes 1 Watt whilst on and transmitting over the cellular link, and close to 0 Watt when switched off. We further assume that the system accommodates 40,000 MTC devices which, as per above figures, allows access to the system in about $1000 \mathrm{~ms}$ and facilitates a duty cycle of about $0.007 \%$. Then, using a small AA battery with about 3 Watt per hour gives a live time of approximately 5 years; in comparison, if no duty cycling is used like in today's mobile phones, life time would be of only 10 hours.

Moreover, in general, the performance of the network becomes worse for all KPIs when the average access interval of MTC is decreased to 15 minutes in Scenario 2 (compared to the 20 minute-case considered in Scenario 1). As it could be expected, the closer the value of the average access interval for MTC devices to that of HTC devices, the lesser the difference that exists between giving priority to one group or the other. 


\section{CONCLuSions \& OPEN IsSUES}

The aim of this paper was to expose the impact of massive MTC deployments onto traditional cellular traffic. Corroborated by rigorous system level simulations, we clearly showed that - under proper design - the impact of MTC onto HTC in the access mechanisms of LTE/LTE-A is negligible as long as HTC are given always priority to request access to the network. We have demonstrated that the duty cycle of MTC devices is indeed sufficiently low to facilitate years-long operation with small batteries. Our findings corroborate that LTE-A service providers will be able to support massive MTC systems without notable impact onto current HTC traffic. However, several important design challenges remain which mainly pertain to the improvement of MTC traffic itself:

Priority class definition and group management for MTC and HTC: The different requirements posed by HTC and MTC need to be mapped and quantified. It is necessary to define the requirements of newly emerged applications for MTC and create groups of applications with common requirements. This will allow for a later particular optimization of the communication networks and a proper group management policy between HTC and MTC. So far, most of research works deal with MTC and HTC as two big groups of applications; however, more granular definition of sub-types of requirements is needed.

Dynamic resource allocation between MTC and HTC: The resources in networks are shared by HTC and MTC devices dynamically. The resources can be initialized to HTC and MTC devices respectively according to the specified rule at the stage of network initialization. When the network detects or predicts in advanced that the network is overloaded by the excessive access attempts caused by the huge number of MTC devices, it dynamically allocates more RACH resources for MTC devices. One of the challenges in this case is when and how to make the adjustment decision. Also, it is still limited by the total amount of available resources.

Separated resources for MTCs in LTE-A: When the same resources are reused by the MTC and HTC devices, the congestion problem can not be completely avoided. Therefore, separating the resources between HTC and MTC devices is a natural idea to decrease congestion and eliminate effects of MTC over $\mathrm{H} 2 \mathrm{H}$ communications. There are, at least, three ways to achieve such separation of resources. First; to split the radio resources in the same frequency band, e.g., RACH channels, access preambles, radio 
resource blocks, and so on, to HTC and MTC devices, separately. Second; to allocate and out-of-band dedicated frequency band (e.g., below $1 \mathrm{MHz}$ ) to serve MTC devices using LTE technology. And third; to allocate an out-of-band dedicated frequency where an optimized technology, different from LTE, is used for MTC transmissions. The first two ways can take advantage of the existing infrastructure at the cost of decreasing the efficiency of LTE/LTE-A networks for HTC services. In contrast, the third option could optimally separate MTC and HTC and provide MTC with an optimal technology tailored for MTC at the cost of having deploy a completely new technology (infrastructure) which has to coexist with LTE/LTE-A.

Delay improvement: Whilst current access delays are well within 100ms, it does not suffice for a wide range of control applications. R13 of 3GPP ought to envisage design solutions facilitating a much quicker access, in the order of a few $m s$. This could likely be achieved by shortening the messaging sequence outlined in Fig. 2, where the biggest challenge will be to make the procedure backwards compliant.

\section{ACKNOWLEDGMENT}

The work was supported by the China Natural Science Funding (61271183), Program for New Century Excellent Talents in University (NCET-11-0600), National High Technology Research and Development Program of China (2014AA01A705), European Research Projects ADVANTAGE (FP7-607774) and NEWCOM\# (FP7-318306).

\section{REFERENCES}

[1] 3GPP GP-100892, "RACH Capacity Evaluation for MTC," TSG GERAN \#46, 2010.

[2] S. Lien, K. Chen, and Y. Lin, "Toward ubiquitous massive accesses in 3GPP machine-to-machine communications," IEEE Commun. Magazine, vol. 49, no. 4, pp. 66-74, Apr. 2011.

[3] C. Ho, and C. Huang, "Energy-saving massive access control and resource allocation schemes for M2M Communications in OFDMA cellular networks," IEEE Wireless Commun. Lett., Apr. 2012.

[4] K. Lee, S. Kim, and B. Yi, "Throughput comparison of random access methods for M2M service over LTE networks," in IEEE Globecom Workshops, 2011, pp. 373-377.

[5] S. Lien and Kwang-Cheng Chen, "Massive access management for QoS guarantees in 3GPP Machine-to-Machine Communications," IEEE Commun. Lett., vol. 15, no. 3, pp. 311-313, Mar. 2011.

[6] S. Lien, T. Liau, C. Kao, and K. Chen, "Cooperative access class barring for Machine-to-Machine communications," IEEE Trans. on Wireless Commun., vol. 11, no. 1, pp. 27-32, Jan. 2012.

[7] T. Kwon, and J. Choi, "Multi-Group random access resource allocation for M2M devices in multicell Systems," IEEE Wireless Commun. Lett., Apr. 2012.

[8] G. Wang, X. Zhong, S. Mei, and J. Wang, "An adaptive medium mccess control mechanism for cellular based Machine to Machine (M2M) communication”, IEEE International Conference on Wireless Information Technology and Systems (ICWITS), 2010, pp. 1-4.

[9] ETSI TS 102690 V2.0.14 (2013-07) Machine-to-Machine communications (M2M) Functional architecture.

[10] P. Jain, P. Hedman, and H. Zisimopoulos, "Machine-Type-Communications in 3GPP Systems," IEEE Wireless Communications Magazine, vol. 50, no. 11, pp. 28 -35, November 2012.

[11] K. Zheng, F. Hu, W. Xiang, M. Dohler, and W. Wang, "Radio resource allocation in LTE-advanced cellular networks with M2M communications", IEEE Communications Magazine, pp.184- 192, July, 2012.

[12] "Standardization of Machine-type Communications (V0.2.2), December 2013," [Online]. Available: http://www.3gpp.org/ftp/Information/WORK_PLAN/Description_Releases/. 
[13] 3GPP TR 36.912 V11.0.0 (2012-09), "Feasibility study for Further Advancement for E-UTRA," Sept. 2012.

[14] E. Dahlman, S. Parkvall, J. Skld and P. Beming, 3G Evolution: HSPA and LTE for Mobile Broadband, 2nd ed. Elsevier Ltd., Oxford, UK, 2008.

[15] 3GPP TS 36.213 V11.4.0 (2013-09), "Evolved Universal Terrestrial Radio Access (E-UTRA); Physical layer procedures (Release 11)," Sept. 2013.

[16] A. Laya, L. Alonso, and J. Alonso-Zarate, "Is the random access channel of LTE and LTE-A suitable for M2M Communications? A survey of alternatives", IEEE Tutorials and Survey Communications Magazine, vol. 16, no. 1, pp. 4-16, January 2014.

\section{BIOGRAPHY}

Kan Zheng (SM'09) received the B.S., M.S. and Ph.D degrees from Beijing University of Posts\&Telecommunications (BUPT), China, in 1996, 2000 and 2005, respectively, where he is currently a professor. He worked as a researcher in companies including Siemens, Orange Labs R\&D (Beijing), China. His current research interests lie in the field of wireless communications, with an emphasis on heterogeneous networks and M2M/V2V networks.

Suling Ou received her B.S. degree from the School of Information and Communication Engineering, Beijing University\&Posts and Telecommunications (BUPT), China, in 2012. She is currently pursing his M.S. degree in the Key Lab of Universal Wireless Communications, Ministry of Education, BUPT. Her research interests include performance analysis, and resource allocation in wireless networks.

\section{Jesus Alonso-Zarate}

Mischa Dohler (FM'13)now is professor in Wireless Communications at King's College London, member of the Board of Directors of Worldsensing, Fellow and Distinguished Lecturer of the IEEE, and Editor-in-Chief of the Transactions on Emerging Telecommunications Technologies. He is a frequent keynote, panel and tutorial speaker. He has contributed to numerous wireless broadband and IoT/M2M standards, holds a dozen patents, chaired numerous conferences, and published more than 160 refereed transactions, conference papers and books. He has a citation h-index of 33.

Fei Liu received his B.S. and M.S. degree from the School of Information and Communication Engineering, Beijing University\&Posts and Telecommunications (BUPT), China, in 2010 and 2013, respectively. He is currently pursing his Ph.D. degree in Aachen University, Germany. His research interests include performance analysis of wireless networks, resource allocation and scheduling algorithms.

Hua Zhu received his B.S. degree from the School of Information and Communication Engineering, Beijing University\&Posts and Telecommunications (BUPT), China, in 2012. He is currently pursing his M.S. degree in the Key Lab of Universal Wireless Communications, Ministry of Education, BUPT. His research interests include M2M communication, and positioning in wireless networks. 